\title{
Effective thermal conductivity of superfluid helium: laminar, turbulent and ballistic regimes
}

\author{
Michele Sciacca $^{1 *}$, Luca Galantucci ${ }^{2}$ \\ ${ }^{1}$ Dipartimento di Scienze Agrarie e Forestali \\ Università degli Studi di Palermo, Palermo, Italy \\ ${ }^{2}$ School of Mathematics and Statistics \\ Newcastle University, Newcastle upon Tyne NE1 7RU, UK \\ *Email address for correspondence: michele.sciacca@unipa.it \\ Communicated by Vito Antonio Cimmelli and David Jou \\ Received on December 31, 2014. Accepted on August 4, 2015.
}

\begin{abstract}
In this paper we extend previous results on the effective thermal conductivity of liquid helium II in cylindrical channels to rectangular channels with high aspect ratio. The aim is to compare the results in the laminar regime, the turbulent regime and the ballistic regime, all of them obtained within a single mesoscopic formalism of heat transport, with heat flux as an independent variable.

Keywords: Thermal conductivity; Liquid helium; Quantum turbulence; Quantized vortices.

AMS subject classification: 76A25, 76F99, 80A99.
\end{abstract}

\section{Introduction.}

Superfluid helium exhibits peculiarities which are very important in cryogenics, for instance for cooling the big magnets at CERN, to keep at low temperature observational devices in astronautical artificial satellites, and for future applications in the refrigeration of micro-devices.

The properties of superfluid helium to flow through very thin capillaries with very low viscosity, or the high thermal conductivity which does not allow it to boil have been known since the first half of the last century [1-7].

From the point of view of heat transport theory, superfluid helium is the most challenging material, because of its peculiar quantum macroscopic coherent nature which confers to it extraordinary properties, and also because it can remain in its liquid state also in the absolute zero temperature limit, potentially exhibiting turbulent behaviour. Even in the latter situation, helium is surprising because of the quantum properties of the vortices 


\section{Sciacca, L. Galantucci}

in turbulent state. In superfluid helium there are different mechanisms responsible for heat transport, but here we focus our attention to the ones occurring in three very different regimes: laminar, turbulent, and ballistic. The first two ones are the subject of much research, but the ballistic transport is much less known, and it occurs when the mean free path ( $\mathrm{mfp}$ ) of the heat carriers (quasiparticles) is comparable or longer to the smallest size of the channel. The heat carriers in Helium II are phonons and rotons as pointed out by Landau [8], even though in the following we refer to phonons for the sake of simplicity, because at very low temperatures their contribution is higher than that of rotons. The aim of this paper is indeed to pay attention to the ballistic regime and continue the studies begun in $[9,10]$, but without dismissing the other two regimes, i.e. in the framework of heat transfer equations able to deal with the three regimes. Furthermore, the dynamical aspects of heat transport are also very special, because of the appearance of second sound waves (temperaure waves at constant density), and because of the strong interaction between thermal effects and mechanical effects.

The main features of the three mentioned devices are the following ones:

1. Laminar regime: The phonon mean free path $(\mathrm{mfp})$ is short as compared with the radius of the pipe in cylindrical channel or with the smallest size in rectangular channel. Furthermore, the heat-flux value is low enough in such a way that the normal component of helium (carrying the heat flow) is laminar. In cylindrical tubes, the total heat flow $\dot{Q}$ and $\nabla T$ are related by the following expression $[2,3,11,12]$ :

$$
\nabla T=-\frac{8 \eta}{R^{2} S^{2} T}\left(\frac{\dot{Q}}{\pi R^{2}}\right)
$$

where $R$ is radius of the pipe, $\eta$ is the viscosity of the normal component, $S$ the entropy per unit volume, $T$ absolute temperature, $\dot{Q}$ the total heat current across the pipe (namely, $\dot{Q}=\pi R^{2} q, q$ being the local heat flux, or the heat flowing per unit time and unit area), and $\nabla T$ the local temperature gradient along the tube.

For laminar flow through a tube with rectangular cross section, the coefficient relating $\nabla T$ and $\dot{Q}$ in (1) is not longer valid. A theoretical solution which is found in Section 2.2. is

$$
\nabla T=-\frac{12 \eta}{b^{2} S^{2} T\left[1-0.63 \frac{b}{a}\right]}\left(\frac{\dot{Q}}{b a}\right)
$$

where $b$ and $a$ are the two sizes of the rectangular cross section with 


\section{Effective thermal conductivity of He II}

$b \ll a$. The coefficient relating $\nabla T$ and $\dot{Q}$ in Eqs. (1) and (2) are the thermal resistance of the pipe.

2. Turbulent regime: For sufficiently high values of the heat flux, quantized vortices appear and contribute to the thermal resistance [13-17], because of the frictional force between the normal component and the quantized vortices. Thus, the heat transport properties becomes strongly nonlinear, very different from those of the laminar regime. The relation between $\nabla T$ and $\dot{Q}$ for fully-developed turbulence is given by [1,2]

$$
\nabla T=-\left(\frac{C_{1} \rho_{n}}{S^{4} T^{3} \rho_{s}^{3}}\right)\left(\frac{\dot{Q}}{A}\right)^{3}
$$

with $\rho_{n}$ and $\rho_{s}$ as the mass densities of normal component and superfluid component, $C_{1}$ a numerical constant and $A$ is the area of the cross section of the channel ( $A=\pi R^{2}$ for a cylindrical channel and $A=a b$ for a parallelepiped channel). The main part of thermal resistance, in contrast to the purely viscous resistance in Landau regime, is now caused by the friction between the normal component (carrying the heat flow) and the quantized vortex tangle. In a recent paper by Sato et al. [18] more experiments were reported on heat transport in channels filled by superfluid helium, where it was observed that in several circumstances the temperature gradient was proportional to the heat flux to the 3.4 power instead of the 3 power. This adds further challenges to the nature and form of heat transport in this regime. It must be said that, furthermore, the transition between the laminar and the turbulent regime is very complicated, so that between (1) and (3) there is a considerable range of intermediate states not yet completely described.

The rectangular tube was not fully studied in this situation apart from the paper by Ladner and Tough [17].

3. Ballistic regime: The phonon $\mathrm{mfp} \ell$ increases when temperature is lowered (for low temperatures, for example, it behaves as $T^{-4.3}$ [4]), in such a way that for sufficiently low temperatures, it becomes comparable to (or higher than) the smallest size of the pipe (below some $0.7 \mathrm{~K} \ell$ is of the order of $0.5 \mathrm{~mm}$ and for lower temperatures it is still larger) [4, 6]. In this case, the predominant collisions are not the phonon-phonon collisions, but the phonon-walls collisions [4]. Thus, in these situations the walls play a crucial role. The expression relating $\nabla T$ and $\dot{Q}$ in this case is $[4,6]$

$$
\nabla T=-\left(\frac{3}{2 C_{v} v R}\right)\left(\frac{f}{2-f}\right) \frac{\dot{Q}}{\pi R^{2}}
$$




\section{Sciacca, L. Galantucci}

with $C_{v}$ being the phonon specific heat, $v$ being the modulus of the phonon speed, and $f$ is the fraction of phonons undergoing diffuse scattering from the tube walls (in contrast to those undergoing specular scattering). For the latter Whitworth in Ref. [19] found that $f$ is a slowly funtion of the temperature $T$ and $f \approx 0.95$ for $T \in[0.3 \mathrm{~K}, 0.6 \mathrm{~K}]$. The ballistic regime shares a number of aspects with the ballistic regime in nanometric solid systems, but it may be approached from the laminar regime as well as from turbulent regime, which is not the case in solids. Up to now, we do not know the equivalent expression of (4) applied to a rectangular channel. In principle, it should be the same relation (4) with the transversal area $a b$ instead of $\pi R^{2}$, but we have found a proposal in Section 3.1. (see expression (25)).

For the sake of completeness, another interesting mechanism in the heat transport in superfluid helium is the "near wall shock wave", which occurs in the presence of a heat flow from the boundary to the fluid [20,21]. It is important just next to the boundary and it is characterized by a thickness $\delta$, in which temperature decays as the distance from the boundary increases (for temperature $T=1.5 \mathrm{~K}$ the thickness $\delta \approx 10^{-6} \mathrm{~cm}$ ).

Although its importance, in this paper we are mainly interested to the situations where the heat flux applied is parallel to the walls. Thus, the contribution of the boundaries becomes relevant when the transversal dimension of the channel is of the same order of the phonon mfp. In this situation it would be interesting to combine the back scattering due to the collisions phonon-wall and the boundary shock wave, which will be investigated in the next future.

The aim of this paper is to consider the transition between the three regimes illustrated, i.e. laminar, turbulent and ballistic, which can be established in terms of the mutual comparison among the length $L^{-1 / 2}$ (the inter-vortex space), $d$ (the smallest size of the channel) and $\ell$ (the phonon mean free path). In particular, the Landau regime is for $\frac{L^{-1 / 2}}{d} \geq 1$ and $\frac{\ell}{d} \ll 1$; the Gortex-Mellinck regime is for $\ell \ll L^{-1 / 2} \ll d$; and the ballistic regime is for $d \leq \ell \ll L^{-1 / 2}$. In particular, special attention is paid to the ballistic regime in parallelepiped devices.

The paper is organized as follows. In Section 2 we consider the equations for the heat flow inside channel with rectangular cross section and we calculate the heat flux profile in the laminar case. In section 3 we consider the influence of the wall when the phonons $\mathrm{mfp}$ is comparable or longer than the smallest size of the channel and some comments on the appearance of quantized vortices. In Section 4 we study the effective thermal conductivity in the presence of quantized vortex lines and slip condition on the 


\section{Effective thermal conductivity of He II}

wall caused by the phonon scattering; and finally Section 5 is devoted to conclusion and comments.

\section{Heat flow along a channel with rectangular transversal section}

In this section we derive the effective thermal conductivity of He II in the laminar regime inside a channel of rectangular cross section with transversal sizes $-\frac{a}{2}<y<\frac{a}{2}$ and $0<z<b$, and length $l$ along the $x$ direction. We describe heat transport in terms of Extended Thermodynamics [22,23] when superfluid helium is globally at rest. In terms of the two-fluid model, the motion of the normal component is compensated by an opposite flow of the superfluid component, in such a way that the net velocity of the total system vanishes, i.e., there is no net mass flow. This means that $\rho_{s} \bar{v}_{s}+\rho_{n} \bar{v}_{n}=0$, where $\bar{v}_{s}$ and $\bar{v}_{n}$ are the average velocities of the superfluid and normal components on the transversal section of the tube, and $\rho_{s}$ and $\rho_{n}$ their density, respectively [13-16].

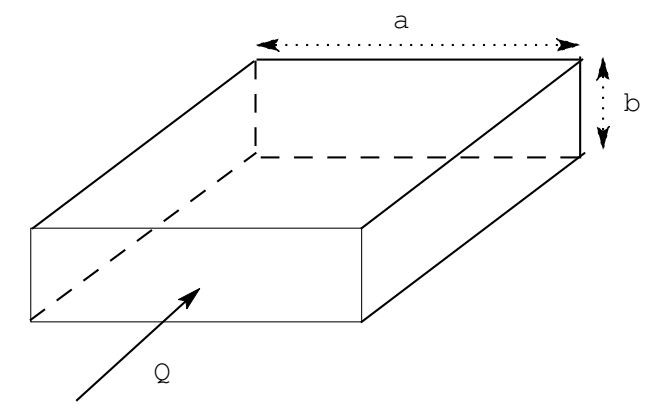

Figure 1. Geometry of the channel being considered with the applied heat flux orthogonal to rectangular section (with size $a$ and $b$ ).

\subsection{Equations for heat transport in laminar flow}

In the one-fluid model of liquid helium II it is rather natural to choose as fundamental fields the mass density $\rho$, the velocity $\mathbf{v}$, the absolute temperature $T$, and the local heat flux q. The dynamical equations in the stationary situation, neglecting the nonlinear terms, for zero net mass flow are [24,25] 


$$
\left\{\begin{array}{l}
\nabla \cdot \mathbf{v}=0 \\
\nabla \cdot \mathbf{q}=0 \\
\nabla p-\eta \nabla^{2} \mathbf{v}-\frac{1}{S T} \eta \nabla^{2} \mathbf{q}=0 \\
\lambda_{1} \nabla T-\frac{\lambda_{1}}{S} \eta \nabla^{2} \mathbf{v}-\frac{1}{S^{2} T} \eta \lambda_{1} \nabla^{2} \mathbf{q}=\sigma^{q}
\end{array}\right.
$$

where $S$ is the entropy per unit volume, $\sigma^{q}$ is the production term of the heat flux, $p$ is the pressure, and $\lambda_{1}$ and $\eta$ can be interpreted as the heat conductivity and the shear viscosity when applied to a classical fluid [24]. The thermal conductivity $\lambda_{1}$ is related to the velocity of second sound $w_{2}$ by the relation $\zeta:=\lambda_{1} / \tau_{1}=w_{2}^{2} \rho C_{v}$, where $C_{v}$ is the constant volume specific heat, and $\tau_{1}$ the relaxation time of the heat flux. Both $\tau_{1}$ and $\lambda_{1}$ are very high in superfluid helium, but their ratio is finite. In these equations, the time derivatives of the corresponding quantities have been neglected because we are interested in steady state situations. Note that the lefthand side of the second equation is the divergence of the flux of the heat flux (second order heat flux). Anyway, it must be said that consideration of the time derivative of the heat flux in the non-steady version of equations (5) is able to to describe in a direct way the second sound.

After some trivial manipulations, Eqs. (5)b and (5)d, respectively, become

$$
\begin{aligned}
& \nabla p-\eta \nabla^{2}\left(\mathbf{v}+\frac{1}{S T} \mathbf{q}\right)=0, \\
& -S \nabla T+\nabla p=-\frac{S}{\lambda_{1}} \sigma^{q} .
\end{aligned}
$$

where we can neglect the contribution $\nabla^{2} \mathbf{v}$ because in Ref. [10] it was seen that it does not contribute to the full heat flow accross a transversal section of the channel in counterflow experiments.

In this section we assume the simplest expression for $\sigma^{q}$, namely $\sigma^{q}=$ $-\mathbf{q}$, while a more general assumption is required to take into account of the presence of vortices $[10,26,27]$.

When $\lambda_{1}$ is high enough, as it is experimentally observed, Eqs. (6a) and (6b) become

$$
\begin{aligned}
& \nabla p-\frac{\eta}{S T} \nabla^{2} \mathbf{q}=0, \\
& S \nabla T-\nabla p=0 .
\end{aligned}
$$

where we recover the famous London's equation (the last equation) relating the pressure gradient and the temperature gradient. 


\section{Effective thermal conductivity of He II}

\subsection{Heat flow through the rectangular channel}

Now, let's set up our concrete problem. Let's assume that the heat flux has the form $\mathbf{q}(y, z)=(q(y, z), 0,0)$ where $y, z$ are the transveral axes and $x$ is the longitudinal coordinate along the channel, and that the heat flux on the wall is also zero. Furthermore, pressure and temperature depend only on the $x$-axis. Thus equation $(7 \mathrm{a})$ becomes

$$
\left[\frac{\partial^{2}}{\partial y^{2}}+\frac{\partial^{2}}{\partial z^{2}}\right] q-\frac{S T}{\eta} \nabla p=0 .
$$

The solution of this partial differential equation is a classical problem in hydrodynamic flow when $q$ is the velocity of the fluid flowing in a channel with the same geometry as considered in this section. The solution is the Poiseuille flow [28]

$$
q(y, z)=-\frac{4 b^{2}}{\pi^{3}} \frac{S T}{\eta} \sum_{n, \text { odd }}^{\infty} \frac{1}{n^{3}}\left[1-\frac{\cosh \left(n \pi \frac{y}{b}\right)}{\cosh \left(n \pi \frac{a}{2 b}\right)}\right] \sin \left(n \pi \frac{z}{b}\right) \nabla p .
$$

The integrated flow across a transversal section is

$$
\dot{Q}=2 \int_{-a / 2}^{a / 2} d y \int_{0}^{b} q(y, z) d z=-\frac{b^{3} a}{12} \frac{S T}{\eta}\left[1-\sum_{n, \text { odd }}^{\infty} \frac{1}{n^{5}} \frac{192}{\pi^{5}} \frac{b}{a} \tanh \left(n \pi \frac{a}{2 b}\right)\right] \nabla p,
$$

For $b / a \rightarrow 0$ (i.e. for thin and wide channels) it becomes

$$
\dot{Q}=-\frac{b^{3} a}{12} \frac{S T}{\eta}\left[1-0.63 \frac{b}{a}\right] \nabla p .
$$

By means of (7b), when $\nabla p$ is expressed in terms of $\nabla T$, expression (11) becomes

$$
\dot{Q}=-\frac{b^{3} a}{12} \frac{S^{2} T}{\eta}\left[1-0.63 \frac{b}{a}\right] \nabla T
$$

and hence the effective thermal conductivity of the superfluid is

$$
K_{e f f} \equiv-\frac{\dot{Q}}{a b} \frac{1}{\nabla T}=\frac{b^{2} S^{2} T}{12 \eta}\left[1-0.63 \frac{b}{a}\right] .
$$

Note that for a channel with infinite parallel plates the second term in the square brackets in the above expressions has to be removed, hence the heat flux acrossing the transversal section of width $a$ and thickness $b$ is [9]

$$
\dot{Q}=-\frac{b^{3} a}{12} \frac{S^{2} T}{\eta} \nabla T
$$


while the effective thermal conductivity is

$$
K_{\text {eff }}=\frac{b^{2} S^{2} T}{12 \eta} .
$$

The reciprocal of this quantity is the thermal resistance per unit length and transversal area. This expression may be compared with the more well-known thermal conductivity of superfluid helium in laminar flow in cylindrical tube of radius $R$, which is [10]

$$
K_{e f f}=\frac{R^{2} S^{2} T}{8 \eta} .
$$

It is seen that for $b \longrightarrow 0$ in (15), $K_{\text {eff }}$ tends to zero as $b^{2}$. Note the same behaviour for $R \longrightarrow 0$ in (16) in a cylindrical channel. However, when compared with experiments (see expression (4)), this reduction is too strong, as it is observed that $K_{\text {eff }}$ tends to zero as $b$ (or $R$ ), rather than as $b^{2}$ (or $R^{2}$ ). This is the reason why (15) is not applicable when $b$ becomes of the order of phonon mfp.

\section{The ballistic regime}

\subsection{Equation and heat profile}

In the ballistic regime, namely when the mfp of the phonon becomes comparable with the size of the system, collision with the surface become very relevant, and even dominant, in setting the thermal resistance. Inspiring in the so-called phonon hydrodynamics in the rarefied regime, one admits a heat flux slip flow along the walls, instead of the non slip condition. For this reason we choose

$$
q_{w}=-C \ell\left(\frac{\partial q(y, z)}{\partial z}\right)_{z=b}
$$

where $C$ is a non-negative parameter, which in kinetic theory of rarefied gases ma be identified as $C=\frac{2-f}{f}[4,6]$. More arguments on the interesting subject of no-slip condition can be find in Ref. [29,30]. Note that the same condition (17) also holds in the wall $z=0$, but with the derivative evaluated in the opposite direction, i.e. always pointing towards the inner of the channel.

In this case the solution of the system (8) is again the solution (9) but with a finite integration constant due to the slip condition on the wall, namely

$$
q(y, z)=-\frac{4 b^{2}}{\pi^{3}} \frac{S T}{\eta} \sum_{n, \text { odd }}^{\infty} \frac{1}{n^{3}}\left[1-\frac{\cosh \left(n \pi \frac{y}{b}\right)}{\cosh \left(n \pi \frac{a}{2 b}\right)}\right] \sin \left(n \pi \frac{z}{b}\right) \nabla p+q_{w}
$$




\section{Effective thermal conductivity of He II}

where $q_{w}$ can be interpreted as the proposed heat flux on the wall (17).

Inserting the solution (9) in the slip condition (17) we obtain the solution

$$
q(y, z)=-\frac{4 b^{2}}{\pi^{3}} \frac{S T}{\eta} \sum_{n, o d d}^{\infty} \frac{1}{n^{3}}\left[1-\frac{\cosh \left(n \pi \frac{y}{b}\right)}{\cosh \left(n \pi \frac{a}{2 b}\right)}\right]\left[\sin \left(n \pi \frac{z}{b}\right)+\frac{n \pi}{b} C \ell\right] \nabla p .
$$

As above, let's calculate the integrated flow across a transversal section (20)

$$
\dot{Q}=-\frac{b^{3} a}{12} \frac{S T}{\eta}\left[1+\frac{6 C \ell}{b}-96 \frac{1}{a} \sum_{n, \text { odd }}^{\infty}\left(\frac{C \ell}{\pi^{3}} \frac{1}{n^{3}}+\frac{2 b}{\pi^{5}} \frac{1}{n^{5}}\right) \tanh \left(n \pi \frac{a}{2 b}\right)\right] \nabla p,
$$

which for $b / a \rightarrow 0$ (i.e. for thin and wide channels) becomes

$$
\dot{Q}=-\frac{b^{3} a}{12} \frac{S T}{\eta}\left[1-0.63 \frac{b}{a}+6 \frac{C \ell}{b}-3.26 \frac{C \ell}{a}\right] \nabla p .
$$

The total heat flow $\dot{Q}$ in terms of the $\nabla T$ can be written by means of (7b)

$$
\dot{Q}=-\frac{b^{3} a}{12} \frac{S^{2} T}{\eta}\left[1-0.63 \frac{b}{a}+6 \frac{C \ell}{b}-3.26 \frac{C \ell}{a}\right] \nabla T
$$

and hence the effective thermal conductivity defined as

$$
K_{\text {eff }}=-\frac{\dot{Q}}{a b} \frac{1}{\nabla T}=\frac{b^{2}}{12} \frac{S^{2} T}{\eta}\left[1-0.63 \frac{b}{a}+6 \frac{C \ell}{b}-3.26 \frac{C \ell}{a}\right]
$$

Note that for channel with infinite plates we recover the same results obtained in [9] (see equation (4.9)). In the case of a very small distance between plates then the wall effects are emphasized and expression (22) can be written

$$
\nabla T=-\frac{12 \eta}{b^{2} S^{2} T C \ell\left[6 \frac{1}{b}-3.26 \frac{1}{a}\right]} \frac{\dot{Q}}{b a}
$$

which for high value of $a$ becomes

$$
\nabla T=-\frac{2 \eta}{b S^{2} T C \ell}\left(\frac{\dot{Q}}{b a}\right)
$$

similar to the one obained for cylindrical channel in [9], namely

$$
\nabla T=-\frac{2 \eta}{R S^{2} C T \ell}\left(\frac{\dot{Q}}{\pi R^{2}}\right)
$$




\section{Sciacca, L. Galantucci}

Expression (26) becomes (4) with the identifications proposed in [9], namely $\eta=\frac{1}{5} \rho_{n} v \ell$ and $S^{2} T=\frac{g \rho_{n} v^{2} C_{v}}{3}$ and $C=\frac{2-f}{f}$ with $g=4 / 5$. Using the same identifications in the expression (24) with high value of $a$, we obtain

$$
\nabla T=-\frac{3}{2} \frac{1}{b v C_{v}}\left(\frac{f}{2-f}\right)\left(\frac{\dot{Q}}{b a}\right)
$$

which is the equivalent to (4), experimentally proposed in [4].

\subsection{Role of heat slip flow on the transition to turbulence}

In the previous paragraph we have computed the slip heat flow along the walls in the ballistic regime, and its role on the effective thermal conductivity. Here, we explore its role on the appearance of the first quantized vortex and on the transition to turbulence. Such influence is due to the modification of the velocity profiles. If $\dot{V}_{n}$ and $\dot{V}_{s}$ be the total volume flow rate of normal and superfluid components of superfluid helium, related to the average velocities by $\dot{V}_{n}=a b \bar{v}_{n}$ and $\dot{V}_{s}=a b \bar{v}_{s}$. Counterflow imposes that $\rho_{n} \dot{V}_{n}=-\rho_{s} \dot{V}_{s}$ and hence the total heat flow is $\dot{Q}=S T \bar{v}_{n} a b=S T \dot{V}_{n}$. The same arguments are of course valid for cylindrical channel but with the area $\pi R^{2}$ in place of $a b$.

Let's first consider the heat flux flowing through two infinity plates. This means neglecting all the negative contributions in the expression (21). Thus, the total heat flow accross a channel of thickness $b$ and width $a$ is given by

$$
\dot{Q}=S T \dot{V}_{n}=-\frac{b^{3} a}{12} \frac{S T}{\eta}\left[1+6 \frac{C \ell}{b}\right]
$$

as also obtained in [9] (see equation (4.8)). Expression (28) is related to the maximum value of heat flux $q_{\max }=q(z)_{\mid z=b / 2}$ inside the channel by

$$
\dot{Q}^{(s)}=\frac{2}{3} a b\left(1+\frac{2 C \ell}{b+4 C \ell}\right) q_{\max }^{(s)},
$$

where the superscript "s" stands for "slip", while for the non slip condition

$$
\dot{Q}^{(n s)}=\frac{2}{3} a b q_{\max }^{(n s)}
$$

where the superscript " $n s "$ stands for "no slip". 


\section{Effective thermal conductivity of He II}

For a given heat flow $\dot{Q}$, what we note is that $q_{\max }^{n s} \geq q_{\max }^{s}$. Indeed,

$$
\dot{Q}^{(n s)}=\dot{Q}^{(s)} \Rightarrow q_{\max }^{(n s)}=\left(1+\frac{2 C \ell}{b+4 C \ell}\right) q_{\max }^{(s)} \geq q_{\max }^{(s)}
$$

The same arguments can be also applied to cylindrical channel of radius $R$. Indeed, by means of the results of the paper [9] we know that the relation between the applied total heat flux $\dot{Q}$ and the value of the maximum heat flux (at the axis of the channel) with and without slip condition are

$$
\dot{Q}^{(s)}=\frac{\pi R^{2}}{2}\left(1+\frac{2 C \ell}{R+2 C \ell}\right) q_{\max }^{(s)}
$$

and for non slip condition

$$
\dot{Q}^{(n s)}=\frac{\pi R^{2}}{2} q_{\max }^{(n s)}
$$

For a given heat flow $\dot{Q}$ in a cylindrical channel we obtain

$$
\dot{Q}^{(n s)}=\dot{Q}^{s} \Rightarrow q_{\max }^{(n s)}=\left(1+\frac{2 C \ell}{R+2 C \ell}\right) q_{\max }^{(s)} \geq q_{\max }^{(s)}
$$

From a qualitative point of view, the first vortices will arise when the available vorticity is equal or slightly higher than the quantum vorticity $\kappa=h / m$ ( $h$ being the Planck's constant and $m$ the helium atomic mass), as discussed in [31]. On the other side, the available vorticity will be of the order of $\left.\left(\rho_{n} / \rho\right)\right)\left|\mathbf{v}_{n}-\mathbf{v}_{s}\right|_{\max } 2 \pi(b / 2), b$ being the separation between plats, or the diameter of a cylindrical channel. Equating these two quantities, a critical quantum Reynolds number appears. The quantum Reynolds number is $R e_{q}=\frac{V_{n s} b}{\kappa}$, with $\kappa$ instead of the kinematic viscosity $\nu$ of the classical Reynolds number, and $V_{n s}=\left|\mathbf{v}_{n}-\mathbf{v}_{s}\right|$ [31]. Quantum Reynolds number can also be written in terms of the heat flux $q$ by means of $q=S T V_{n s}$, namely $R e_{q}=\frac{q b}{S T \kappa}$.

In [31] it has seen that the appearance of the first vortex occurs for $R e_{q} \approx \frac{1}{\pi} \frac{\rho}{\rho_{n}} \ln \left(\frac{b}{2 a_{0}}\right)$, with $a_{0}$ the size of the core of the vortex. According to the above results, the presence of the slip condition modifies the maximum value of the heat flux inside the channel and it becomes smaller than the corresponding one with zero-slip condition. This means that we have to apply a higher $\dot{Q}$ for the appearance of the first vortex in such a way that 


\section{Sciacca, L. Galantucci}

$q_{\max }^{(s)}$ reaches the critical value. In terms of the quantum Reynolds number for infinite plates

$$
R e_{q}^{(n s)}=\frac{q_{\max }^{(n s)} b}{S T \kappa}=\left(1+\frac{2 C \ell}{b+4 C \ell}\right) \frac{q_{\max }^{(s)} b}{S T \kappa} \geq \frac{q_{\max }^{(s)} b}{S T \kappa}=R e_{q}^{(s)} .
$$

and analogously for a cylinder channels. From a qualitative point of view, the walls delay the appearance of vortices by a factor $\left(\frac{2 C \ell}{b+4 C \ell}\right)$ for infinite plates and by a factor $\left(\frac{2 C \ell}{R+2 C \ell}\right)$ for a cylindrical channel. This delay could occur also for the other critical Reynolds numbers for the appearance of the TI turbulence and TII turbulence in cylindrical channel as well as for for the appearance of TIII turulence in rectangular cross section channel with high aspect ratio.

\section{Turbulent regime (Gorter-Mellinck)}

In this Section we consider the presence of a vortex tangle in order to find out how the heat conducibility changes when quantum vortices are distributed all over the channel. It is well known that quantum vortices appear when the applied heat flux is higher than a critical value $[13,14,16$, 32]. Tough and coworker studied turbulence and the transition to turbulence in superfluid helium for a lot of channel size and shape. They found out a further transition in the turbulente regime for cylindrical channel which distinguishes turbulence in two kind of turbulence (state TI and state TII) [32]. In a rectangular channel with high aspect ratio instead Ladner and Tough found just one state of turbulence, which they called TIII [17].

In the turbulent regime we must consider an additional equation for the vortex line $L$, which also takes into account the size of the channel. For the sake of simplicity we consider here the same equation used in Ref. [10]

$$
\frac{d L}{d t}=-\beta_{v} \kappa L^{2}+\left[\alpha_{0} v_{n s}-\omega^{\prime} \beta \frac{\kappa}{d}\right] L^{3 / 2}
$$

where coefficients $\alpha_{0}$ and $\omega^{\prime}$ are functions of $v_{n s} d / \kappa, d$ is the radius of the cylindrical channel or the minimum value between the two sizes of the channel $d=\min \{a, b\}$ in rectangular channel (which becomes $b$ in the high aspect ratio situation considered in this paper).

Equation (36) was proposed on dimensional grounds taking into account the lowest size in the channel compared to the mean distance between vortex lines $L^{-1 / 2}$, namely the ratio $\frac{L^{-1 / 2}}{d}$. Indeed, in the equation (36) it was considered only the first two terms in the expansion of distruction 


\section{Effective thermal conductivity of He II}

$\operatorname{term} \beta \kappa L^{2}$, which is $\beta \kappa L^{2}\left(1+\omega^{\prime} \frac{L^{-1 / 2}}{d}+\omega^{\prime \prime}\left(\frac{L^{-1 / 2}}{d}\right)^{2}+\ldots\right)$, but more terms could be considered as well as in the production term $\alpha_{1} q L^{3 / 2}$ [33].

The full system in the steady state then becomes

$$
\begin{aligned}
& \nabla p-\frac{1}{S T} \eta \nabla^{2} \mathbf{q}=0, \\
& S \nabla T-\nabla p+\frac{K S L}{\zeta} q=0, \\
& -\beta_{v} \kappa L^{2}+\left[\alpha_{0} v_{n s}-\omega^{\prime} \beta \frac{\kappa}{d}\right] L^{3 / 2}=0
\end{aligned}
$$

The third equation (37c) can be also written in terms of the heat flux $q$ by means of $q=\left(\rho_{s} / \rho\right) S T v_{n s}$.

Equation (37b) has the steady state solutions

$$
L=0 ; \quad L^{1 / 2}=\gamma \frac{\rho}{\rho_{s} S T} q-\frac{\omega^{\prime}}{d} .
$$

where $\gamma=\frac{\alpha_{0}}{\beta \kappa}$. The second solution is stable for $q>q_{c 1}=\frac{\rho_{s} S T}{\rho} \frac{\beta \kappa \omega^{\prime}}{\alpha_{0} d}$, and in Ref. [34] it is seen that it has two different regimes, namely a TI turbulence and TII turbulence flow. In a channel with a rectangular cross section with high aspect ratio, instead, just one status was revealed, which suggests that the turbulence in these kind of channels is simpler than the one in the cylindrical channels.

Thermal counterflow in high-aspect ratio rectangular tubes exhibits only one turbulent state (also named TIII) with the coefficient $\gamma$, named $\gamma_{3}(T)$, comparable with the $\gamma_{2}(T)$ coefficient, which is the value of $\gamma$ in the TII turbulence in cylindrical tubes. From Yarmchuck and Glaberson's results (applied to wide channel) [35] and Ladner and Tough's results (applied to narrow channel) [17] it was stated that $\gamma_{3}(T)$ does not depend on the tube size.

The expression of $\gamma$, instead, depends on the geometry of the channel by a ratio $D / d$, where $D$ is the hydraulic diameter $(D=4 A / P$ with $A$ the area and $P$ the perimeter of the transversal section of the channel) and $d$ is the radius $R$ in the cylindrical channel and the size $b$ in the rectangular channel. In the former $D / d=1$, while in the latter $D / d>1$.

Below we distinguish two main situations: $\ell \ll L^{-1 / 2} \ll d$ and $L^{-1 / 2} \ll d \leq \ell$. In both cases, on the grounds of past numerical simulations [36,37], we assume that the vortex line density $L$ is constant on any transversal section and, therefore, we stress that $L$ should be considered as the homogeneous equivalent vortex-line density. 


\section{Sciacca, L. Galantucci}

\subsection{Case $\ell \ll L^{-1 / 2} \ll d$}

The first equation (37a) is not changed and the solution is still expression (9) because we are in the same conditions as the previous section. The heat flow accross a transversal section is still the expression (10), which can be simplified in (11).

Integrating the second equation (37b) on the trasversal section one obtains

$$
a b S \nabla T-a b \nabla p+\frac{K S L}{\zeta} \dot{Q}=0 .
$$

where $L$ is mean value of the vortex line density on the transversal section of the channel. Expressing the previous equation in terms of $\nabla p$ and substituting it in the expression (9), then one can obtain

$$
\nabla T=-\left(\frac{12 \eta}{S^{2} T b^{2}\left[1-0.63 \frac{b}{a}\right]}+\frac{K L}{\zeta}\right)\left(\frac{\dot{Q}}{a b}\right)
$$

and hence the thermal conductivity

$$
K_{e f f}=\frac{1}{\left(\frac{12 \eta}{S^{2} T b^{2}\left[1-0.63 \frac{b}{a}\right]}+\frac{K L}{\zeta}\right)} .
$$

But $L$ is itself a function of $\dot{Q}$ when $\dot{Q}$ is high. Expression (40) is the one used by Ladner and Tough for their studies. As one can see in their paper, this expression fits very well the experimental data for different temperatures and channel sizes.

\subsection{Case $L^{-1 / 2} \ll d \leq \ell$}

Now, we consider the case in which the phonon mean free path is comparable to the sizes of the channel and much longer than the main distance between quantized vortices. This condition requires a no-null value of the heat flux $q(y, z)$ on the wall. The dynamical equations are still (8), but the solution of the equation (37a) to be considered is the solution (19) and the corresponding heat flux accross the transversal section (20) or (21).

Integrating the second equation (37b) on the trasversal section we obtain (39), where we can substitute the expression (20) or (21) of $\dot{Q}$. The result is

$$
\nabla T=-\left(\frac{1}{b^{2} A}+\frac{K S L}{\zeta}\right)\left(\frac{\dot{Q}}{a b}\right)
$$




\section{Effective thermal conductivity of He II}

where $A=\frac{S T}{12 \eta}\left[1+\frac{6 C \ell}{b}-96 \frac{1}{a} \sum_{n, o d d}^{\infty}\left(\frac{C \ell}{\pi^{3}} \frac{1}{n^{3}}+\frac{2 b}{\pi^{5}} \frac{1}{n^{5}}\right) \tanh \left(n \pi \frac{a}{2 b}\right)\right]$ for the solution (20) and $A=\frac{S T}{12 \eta}\left[1-0.63 \frac{b}{a}+6 \frac{C \ell}{b}-3.26 \frac{C \ell}{a}\right]$ for the solution (21).

The effective thermal conductivity is then given by

$$
K_{e f f}=\frac{1}{\left(\frac{1}{b^{2} A}+\frac{K S L}{\zeta}\right)}
$$

Note that expression (40) for the total heat flux and (41) for thermal conductivity are not longer valid when the scattering of phonon on the wall become relevant. Here, we take into account two opposite contribution to the total heat flux and to $K_{\text {eff }}$ : from one hand the presence of the scattering of phonons (slip condition) raises the value of $K_{\text {eff }}$ and the dissipative effect of the vortex lines which decreases $K_{\text {eff }}$.

\section{Discussion and Conclusion}

In this paper we have considered the effective thermal conductivity in a counterflow channel with rectangular cross section with high aspect ratio. It is interesting to note that the model is able to describe not only the three regimes (laminar, turbulent and ballistic), but it also describes the transition among them. In [9] we considered the effective thermal conductivity in cylindrical channel in the same regimes considered here, but now we pay more attention to the wall effects on the transition to quantum turbulence. As in [9] we find that when the distance $b$ between the plates (or the radius $R$ in the cylindrical channel) is small enough then the effective thermal conductivity is not proportional to $b^{2}$ (or $R^{2}$ ), as one erroneously could think from expression (13). Indeed, in Section 3.1. we obtain expression (23) which is proportional to $b$ for very small value of $b$. Expression (23) takes into account the transition between laminar and ballistic regimes, and for $b$ very small we find expression (25), which is equivalent to the experimental proposal in the ballistic regime in cylindrical channel $[4,6]$. Further studies are required to handle the so called "near wall shock wave" in the proximity of the hot walls $[20,21]$.

In Section 3.2. we have paid attention to an argument which has not been considered yet, at least to our knowledge. Indeed, when the effects of the walls are not neglected then all the arguments, referring to heat flux profile, transition to quantum turbulence and so on, have to be reconsidered. The result is that when the mfp of the phonons becomes comparable to the 


\section{Sciacca, L. Galantucci}

smallest size of the channel, then the heat flux has a slip condition on the walls, so that the maximum value of the heat flux $q$ inside the channel is lowered (the profile is more flattened). It implies that more external heat flux $\dot{Q}$ is necessary in such a way that the maximum value of the heat flux becomes higher than the critical value for the appearance of the first quantized vortex line.

In Section 4 we have considered the presence of quantized vortices in the channel and their influence on the effective thermal conductivity. For it, a further equation has been necessary (equation (36)), which we have already proposed in [10], and we have used here for the sake of uniformity. Unfortunately, there are not enough papers dealing with parallelepiped counterflow channels. In [17] Ladner and Tough consider four long channels with a rectangular $10 b \times b$ cross section with $b=0.0098 \mathrm{~cm}, b=0.0047 \mathrm{~cm}, b=0.0032$ $\mathrm{cm}$ and $b=0.0091 \mathrm{~cm}$ in the range of temperatures $1.2 \mathrm{~K}$ to $1.8 \mathrm{~K}$. According to their results, they found only one state of quantum turbulence (the TIII turbulent regime), which reveals that rectangular channel is simpler than quantum turbulence in cylindrical channel, where two kinds of turbulence have been detected [32,34].

Our expression(40) can be also obtained by means of the two-fluid model [38-41] (as also found in [9,10]), and indeed it coincides with that taken by Ladner and Tough, apart from the expression of the steady vortex line density (their equation (25)), which they approximated within $2 \%$ by the expression

$$
L_{0}^{1 / 2}=1.03 \gamma\left(V-0.36 V_{c}\right)
$$

where $V=\frac{\rho}{\rho_{s} S T} q$ is the counterflow velocity, $\gamma=\frac{\alpha_{0}}{\beta \kappa}$ and $V_{c}$ is the critical counterflow velocity for the appearence of quantum turbulence. Expression (44) is practically our expression (38b), which is also able to describe the transition between the turbulent TI regime and the turbulent TII regime in the cylindrical channel [10].

In the last Section 4.2. we take into account the presence of the walls. What we find is that the effective thermal conductivity has further terms from the slip condition, apart from the ones coming from the presence of quantum turbulence and laminar flow. In this respect, in their experiments Ladner and Tough do not consider any contribution from the walls. They instead adopt the stringent condition that the measured laminar temperature difference must agree with equation (2) over a wide range of temperatures, and any further discrepancy of their results from this formula or, more in general, from formula (40) are considered in details.

The arguments of this paper open the interest to further studies on the 


\section{Effective thermal conductivity of He II}

refrigeration of small devices located in a parallelepiped channel with high aspect ratio where a cryogenic fluid flows.

\section{Acknowledgements}

The authors acknowledge the highligthing discussions with Prof. David Jou from the Universitàt Autònoma de Barcelona. The authors acknowledge the financial support by the National Group of Mathematical Physics (GNFM-INdAM) and the Università di Palermo (under Grant Nos. Fondi 60\% 2012 and Progetto CoRI 2012, Azione d). M.S. acknowledges the hospitality of the "Group of Fisica Estadistica of the Universitàt Autònoma de Barcelona". L. G.'s work is supported by Marie Curie - COFUND action through Grant N. 7745104 issued by Fonds National de la Recherche, Luxembourg.

\section{REFERENCES}

1. V. Arp, Heat transfer to superfluid and supercritical helium, Journal of Applied Physics, vol. 40, p. 2010, 1969.

2. K. Mendelsohn, Liquid helium, in Encyclopedia of Physics, vol. XV, Berlin: Springer, 1956.

3. S. W. Van Sciver, Helium Cryogenics. Berlin: Springer, second ed., 2012.

4. B. Bertman and T. A. Kitchens, Heat transport in superfluid filled capillaries, Cryogenics, vol. 8, pp. 36-41, 1968.

5. D. Benin and H. J. Maris, Phonon heat transport and Knudsen"s minimum in liquid helium at low temperatures, Physical Review B, vol. 18, pp. 3112-3125, 1978.

6. D. S. Greywall, Thermal-conductivity measurements in liquid ${ }^{4}$ He below 0.7k, Physical Review B, vol. 23, pp. 2152-2168, 1981.

7. H. J. Maris, Dissipative coefficients of superfluid helium, Physical Review A, vol. 7, pp. 2074-2081, 1973.

8. L. D. Landau, Theory of the superfluidity of helium II, Physical Review, vol. 60, no. 4, p. 356, 1941.

9. M. Sciacca, A. Sellitto, and D. Jou, Transition to ballistic regime for heat transport in helium II, Physics Letters A, vol. 378, pp. 2471-2477, 2014.

10. M. Sciacca, D. Jou, and M. S. Mongioví, Effective thermal conductivity of helium ii: from landau to gorter-mellink regimes, Zeitschrift für angewandte Mathematik und Physik, pp. 1-17, 2013.

11. P. Critchlow and R. Hemstreet, Heat transport in superfluid helium in 


\section{Sciacca, L. Galantucci}

wide tubes, Journal of Applied Physics, vol. 40, p. 2675, 1969.

12. L. D. Landau and E. M. Lishitz, Mechanics of fluids. Oxford: Pergamon, 1985.

13. R. J. Donnelly, Quantized vortices in helium II. Cambridge, UK: Cambridge University Press, 1991.

14. C. F. Barenghi, R. J. Donnelly, and W. F.Vinen, Quantized Vortex Dynamics and Superfluid Turbulence. Berlin: Springer, 2001.

15. M. Tsubota, M. Kobayashi, and H. Takeuchi, Quantum hydrodynamics, Physics Report, vol. 522, pp. 191-238, 2013.

16. S. K. Nemirovskii, Quantum turbulence: Theoretical and numerical problems, Physics Reports, vol. 524, pp. 85-202, 2013.

17. D. R. Ladner and J. T. Tough, Temperature and velocity dependence of superfluid turbulence, Physical Review B, vol. 20, pp. 2690-2702, 1979.

18. A. Sato and et al., Temperature dependence of the Gorter-Mellink exponent measured in a channel containing He II, in ADVANCES IN CRYOGENIC ENGINEERING: Transactions of the Cryogenic Engineering Conference-CEC, vol. 823, pp. 387-392, AIP Publishing, 2006.

19. R. Whitworth, Experiments on the flow of heat in liquid helium below 0.7 degrees K, Proceedings of the Royal Society of London. Series A. Mathematical and Physical Sciences, vol. 246, no. 1246, pp. 390-405, 1958.

20. S. Putterman, Superfluid hydrodynamics. Amsterdam: North-Holland Publishing Co., 1974.

21. S. Nemirovskii, V. Koren'kov, and V. Krupitskii, Heat flux through a phase interface in superfluid helium, Journal of Engineering Physics, vol. 47, no. 6, pp. 1413-1419, 1984.

22. D. Jou, J. Casas-Vázquez, and G. Lebon, Extended Irreversible Thermodynamics. Berlin: Springer-Verlag, fourth ed., 2010.

23. I. Müller and T. Ruggeri, Rational Extended Thermodynamics. New York: Springer-Verlag, 1998.

24. M. S. Mongioví, Extended irreversible thermodynamics of liquid helium II, Physical Review B, vol. 48, pp. 6276-6283, 1993.

25. D. Jou, J. Casas-Vázquez, and M. Criado-Sancho, Thermodynamics of Fluids Under Flow. Berlin: Springer, second ed., 2011.

26. D. Jou, G. Lebon, and M. S. Mongiovì, Second sound, superfluid turbulence, and intermittent effects in liquid helium II, Physical Review B, vol. 66, p. 224509 (9 pages), 2002.

27. D. Jou and M. Mongiovì, Description and evolution of anisotropy in superfluid vortex tangles with counterflow and rotation, Physical Review $B$, vol. 74, p. 054509 (11 pages), 2006. 


\section{Effective thermal conductivity of He II}

28. H. Bruus, Theoretical microfluidics. Oxford: Oxford University Press, 2007.

29. D. Jou, G. Lebon, and M. Criado-Sancho, Variational principles for thermal transport in nanosystems with heat slip flow, Physical Review $E$, vol. 82, no. 3, p. $031128,2010$.

30. G. Lebon and P. Dauby, Heat transport in dielectric crystals at low temperature: A variational formulation based on extended irreversible thermodynamics, Physical Review A, vol. 42, p. 4710, 1990.

31. D. Jou and M. Sciacca, Quantum Reynolds number for superfluid counterflow turbulence, in Bollettino di Matematica Pura e Applicata (M. S. Mongioví, M. Sciacca, and S. Triolo, eds.), vol. VI, pp. 95-103, Aracne editrice, 2013.

32. J. Tough, Superfluid turbulence, in Progress of Low Temperature Physics (D. Brewer, ed.), vol. VIII, pp. 133-219, North Holland, 1982.

33. M. S. Mongiovì and D. Jou, Generalization of Vinen's equation including transition to superfluid turbulence, Journal of Physics: Condensed Matter, vol. 17, pp. 4423-4440, 2005.

34. K. P. Martin and J. T. Tough, Evolution of superfluid turbulence in thermal counterflow, Physical Review B, vol. 27, pp. 2788-2799, 1983.

35. E. Yarmchuk and W. I. Glaberson, Thermorotation Effects in Superfluid Helium, Physical Review Letters, vol. 41, no. 8, pp. 564-568, 1978.

36. L. Galantucci, C. F. Barenghi, M. Sciacca, M. Quadrio, and P. Luchini, Turbulent Superfluid Profiles in a Counterflow Channel, Journal of Low Temperature Physics, vol. 162, pp. 354-360, 2011.

37. L. Galantucci and M. Sciacca, Non-classical velocity statistics in counterflow quantum turbulence, Acta Applicandae Mathematicae, vol. 132, no. 1, pp. 273-281, 2014.

38. L. D. Landau, Theory of the Superfluidity of Helium II, Journal of Physics, vol. 5, pp. 71-90, 1941.

39. L. Tisza, Transport phenomena in Helium II, Nature, vol. 141, p. 913, 1938.

40. I. K. I.L. Bekarevich, Phenomenological derivation of the equation of vortex motion in he ii, Soviet Physics JETP, vol. 13, pp. 643-646, 1961.

41. H. Hall and W. Vinen, The rotation of liquid helium ii. ii. the theory of mutual friction in uniformly rotating helium II, Proceedings of the Royal Society of London. Series A. Mathematical and Physical Sciences, vol. 238, no. 1213, pp. 215-234, 1956. 